\title{
Nonalcoholic fatty liver disease among patients with irritable bowel syndrome: Prevalence and contribution to disease severity
}

\author{
Ahmad FA Hasanain ${ }^{1 *}$, Mohamed E Abdel-Rahman ${ }^{1}$, Amany M Ali' ${ }^{1}$ and Sherif M Abdel-Aal ${ }^{2}$ \\ ${ }^{1}$ Department of Tropical Medicine and Gastroenterology, Faculty of Medicine, Assiut University, Egypt \\ ${ }^{2}$ Department of Diagnostic Radiology, Faculty of Medicine, Assiut University, Egypt
}

\begin{abstract}
Background/aim: Irritable bowel syndrome (IBS) and nonalcoholic fatty liver disease (NAFLD) are common diseases in our locality. To our knowledge, no previous studies have investigated the NAFLD among patients with IBS. The aim of this study is to determine the prevalence of NAFLD among patients with IBS and to explore the association of NAFLD with the severity of IBS.
\end{abstract}

Patients and methods: The study included 100 consecutive patients with IBS. The diagnosis of IBS relied on Rome III criteria. For all the study population, clinical evaluation, abdominal ultrasonography, and laboratory investigations (including fasting serum levels of glucose and lipids profile) were conducted.

Results: The mean age of the study population was $47 \pm 12$ years; $49 \%$ of them were males. Moderate/severe IBS was determined among $58 \%$ of the patients. Nonalcoholic fatty liver disease was detected among $74 \%$ of the patients; moderate/severe grade was among $57 \%$ of the patients. The metabolic syndrome (MS) was diagnosed among $19 \%$ of the patients. The independent predictors of moderate/severe IBS were moderate/severe NAFLD and MS.

Conclusion: In conclusion, we detected a high prevalence rate of NAFLD among patients with IBS. The predictors of moderate/severe IBS among our study patients were moderate/severe NAFLD and MS.

\section{Introduction}

Altered bowel habits associated with abdominal discomfort or pain, in the absence of structural and biochemical abnormalities, is defined as Irritable bowel syndrome (IBS), which is estimated to affect up to $23 \%$ of the global population [1,2]. With poorly defined etiology, visceral hypersensitivity, altered gastrointestinal motility, brain-gut interactions, altered fecal microbiota, bacterial overgrowth, post infectious reactivity, carbohydrate malabsorption, food sensitivity, and intestinal inflammation all were accused for the pathogenesis of IBS. According to the Rome III diagnostic criteria, IBS is diagnosed based on the presence of recurrent abdominal pain or discomfort for at least three days per month during the last three months, associated with two or more of the following manifestations: onset associated with a change in stool frequency, onset associated with a change in stool consistency, and improvement with defecation [3]. The severity of IBS can be classified according to its impact on lifestyle [4]. Although some studies found a correlation between obesity and IBS [5,6], there are no studies reporting any link between NAFLD and IBS.

Nonalcoholic fatty liver disease (NAFLD) is considered when there is excessive fat accumulation in hepatocytes, in the absence of specific causes of hepatic steatosis, such as alcohol consumption, with or without necroinflammation and its consequences [7]. Its prevalence among the adult population can reach up to $31 \%$ [8]. The pathogenesis of NAFLD relies on obesity and insulin resistance [9]. Nonalcoholic fatty liver disease may be hypothetically accused for the development of IBS through the inflammatory state (induced by adipokines and free fatty acids) $[10,11]$ responsible for the progression of hepatic steatosis to nonalcoholic steatohepatitis.

Our research hypothesis is that NAFLD may contribute to the severity of IBS through an inflammatory pathway. To our knowledge, no previous studies have investigated the NAFLD among patients with IBS. The aim of this study is to determine the prevalence of NAFLD among patients with IBS and to explore the association of NAFLD with the severity of IBS.

\section{Patients and methods}

\section{Study design}

A hospital-based, cross-sectional study was performed.

\section{Study location}

The study population was recruited from patients attending the outpatient clinics of the Department of Tropical Medicine and Gastroenterology.

*Correspondence to: Ahmad Farooq Alsayed Hasanain, Department of Tropical Medicine and Gastroenterology, Assiut University Hospitals, Assiut, Egypt, Tel: 002-0102-0288-660; E-mail: af.hasanain@outlook.com

Key words: irritable bowel; fatty liver, metabolic syndrome

Received: June 08, 2018; Accepted: June 21, 2018; Published: June 25, 2018 


\section{Study duration}

The study population was recruited during the period from January 2016 to February 2018.

\section{Inclusion criteria}

Our study included 100 patients with IBS, diagnosed according to Rome III diagnostic criteria, based on the presence of recurrent abdominal pain or discomfort for at least three days per month during the last three months, associated with two or more of the following manifestations: onset associated with a change in stool frequency, onset associated with a change in stool consistency, and improvement with defecation [3].

\section{Exclusion criteria}

Patients younger than 18 years and pregnant patients were excluded from the study. In addition, patients with alarming signs such as alimentary tract bleeding, anemia, weight loss, and abdominal masses were not included in the study. Patients with any alcohol intake, those using systemic corticosteroids, and patients infected with hepatitis $\mathrm{C}$ virus (HCV) and/or hepatitis B virus (HBV) were also excluded.

\section{Methods}

Clinical evaluation, imaging studies, and laboratory investigations were conducted for the study population. Body mass index (BMI) was estimated according to the following equation: $\mathrm{BMI}=$ weight (in kilograms)/height (in meters) ${ }^{2}$. Body mass index of $25 \mathrm{~kg} / \mathrm{m}^{2}$ to $<$ $30 \mathrm{~kg} / \mathrm{m}^{2}$ denoted overweight, while that $\geq 30 \mathrm{~kg} / \mathrm{m}^{2}$ defined obesity. Waist circumference was measured at the midpoint between the lower costal margin and the iliac crest. Abdominal ultrasonography (US) and laboratory investigations including estimation of fasting serum glucose level, lipids profile (fasting serum levels of total cholesterol, low density lipoprotein cholesterol (LDL-C), high density lipoprotein cholesterol (HDL-C), and triglycerides), liver chemistry panel, and international normalized ratio, and complete blood count were provided. Glucose intolerance was defined as fasting glucose level of more than 6.1 to less than $7 \mathrm{mmol} / \mathrm{L}$, while fasting glucose level of $7 \mathrm{mmol} / \mathrm{L}$ or more represented diabetes mellitus (DM). Dyslipidemia was defined as one or more of the following: total cholesterol serum level more than 200 $\mathrm{mg} / \mathrm{dL}$, LDL-C serum level more than $130 \mathrm{mg} / \mathrm{dL}$, HDL-C serum level less than $27 \mathrm{mg} / \mathrm{dL}$, and triglycerides serum level of $165 \mathrm{mg} / \mathrm{dL}$ or more.

Diagnosis of NAFLD was based on the following criteria: (1) ultrasonographic findings (hyperechogenecity of the liver parenchyma, i.e., bright liver relative to spleen and right kidney, hepatomegaly, and blurring of vascular margins) [3]; (2) no history of alcohol consumption; (3) no exposure to steatogenic medications; (4) no evidence of viral hepatitis B or C. Abdominal ultrasonography was performed by a single, experienced radiologist using Siemens, Sonoline Siena set (Germany). The grading of hepatic steatosis was according to the following: mild grade when the echogenicity is just increased; grade II: when the echogenic walls of portal vein branches are obscured by the echogenic liver; grade III: when the echogenic liver obscures the diaphragmatic outline [12].

The diagnosis of the metabolic syndrome (MS) was considered if three or more of the following items were present: (1) waist circumference more than $102 \mathrm{~cm}$ for males or more than $88 \mathrm{~cm}$ for females; (2) blood pressure reading of $135 / 85 \mathrm{mmHg}$ or more or receiving therapy for systemic hypertension; (3) high fasting serum levels of glucose ( $\geq 110 \mathrm{mg} / \mathrm{dL}$ ) or receiving therapy for either glucose intolerance or DM; (4) high fasting serum levels of triglycerides ( $\geq 150$ $\mathrm{mg} / \mathrm{dL}$ ) or receiving therapy for hypertriglyceridemia; (5) low fasting serum levels of HDL-C less than $40 \mathrm{mg} / \mathrm{dL}$ for males or less than $50 \mathrm{mg} /$ $\mathrm{dL}$ for females, or receiving therapy for low HDL-C level [13].

\section{Statistical analysis}

Data were analyzed using the Statistical Package for Social Sciences (IBM SPSS Statistics, version 22.0, release 22.0.0.0; IBM Corp, Armonk, New York, US) for Microsoft Windows' (64-bit version). Results were expressed as mean \pm standard deviation or frequency (percentage) as appropriate. The predictors of moderate/severe IBS among the study population with IBS were identified using univariate analyses (Yates' corrected chi-square test or Fischer's exact test). Multivariate analysis (binary logistic regression) was used to assess the independent effect of each predictor. Multivariate analysis included significant factors with $p$ $<0.05$ in the univariate analyses.

The software $G^{\star}$ Power version 3.1.9.2 was used for a post hoc power analysis of the performed chi-square tests. An arbitrary effect size was chosen for the power analysis, which precisely was a Cohen's $\mathrm{w}$ statistic of 0.3 . This value conventionally corresponds to a mediumsized effect. The power achieved was 0.84 .

\section{Ethical considerations}

After approval of the Clinical Research Ethical Committee of Assiut Faculty of Medicine, the study was conducted according to the code of ethics of the World Medical Association (Declaration of Helsinki). A consent certificate was signed by all the study patients, after discussing in detail with the investigators the certificate subjects and the study aim. Participants were clearly informed that refusing to participate in the study will not affect having full benefit of the available medical service. Data confidentiality was respected.

\section{Results}

The demographic, clinical, laboratory, and imaging characteristics of the study population with IBS are shown in Table 1 . The mean age was $47 \pm 12$ years. According to the gender, they were almost equally distributed ( $51 \%$ of the patients were females). Those with risk factors for anxiety were those who are married, divorced, and widowed (78\%) and those who are employed (81\%). The most frequent type of IBS was the constipation predominant one (45\%), while the most frequent grade was the moderate one (43\%). Moderate/severe IBS represented $58 \%$ of the study population. The most frequent metabolic disorder was high BMI (89\%). Using abdominal ultrasonography, NAFLD was diagnosed among $74 \%$ of the study population; the moderate grade was the most frequent one (33\%) and 57\% had moderate/severe NAFLD. Alanine aminotransferase and aspartate aminotransferase levels were normal among all the study population.

Table 2 shows that tobacco smoking, DM, MS (25.9\%, 95\% CI $18.3 \%-34.7 \%$ vs $9.5 \%, 95 \%$ CI $6.1 \%-13.6 \%, p$ 0.009) and moderate/ severe NAFLD $(22.4 \%$, $95 \%$ CI $15.8 \%-31.5 \%$ vs $4.8 \%, 95 \%$ CI $2.7 \%$ $7.9 \%, p 0.001)$ were significantly associated with moderate/severe IBS.

On multivariate analysis of the previously determined factors, MS (OR 3.1, 95\% CI 1.8-54.6, $p$ 0.011) and moderate/severe NAFLD (OR 2.4, 95\% CI 1.3-62.7, p 0.026) were significantly, independently associated with moderate/severe IBS (Table 3). Figure 1 shows the outcome among the study patients with IBS. 


\section{Discussion}

Comparing the study patients with moderate/severe IBS to those with mild IBS, we found significantly more frequent moderate/severe NAFLD among patients with moderate/severe IBS (22.4\% vs. $4.8 \%)$ referring to a potential contribution of the higher grades of NAFLD to more severe grades of IBS. The multivariate analysis has confirmed the association of moderate/severe NAFLD to moderate/severe IBS, versus mild IBS, independent of the other risk factors of IBS. As far as we know, this is the first study to assess the prevalence of NAFLD

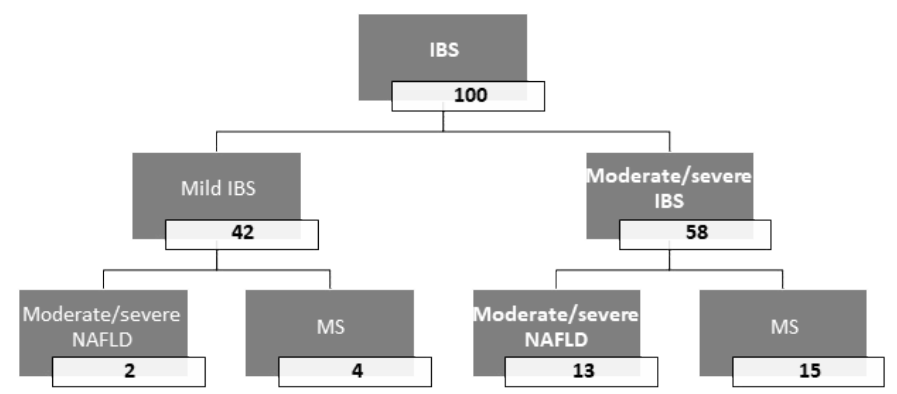

Figure 1. Flow chart of the outcome among the study population with IBS

IBS: irritable bowel syndrome; NAFLD: nonalcoholic fatty liver disease; MS: metabolic syndrome

Table 1. Demographic, clinical, laboratory, and imaging characteristics of the study population with IBS $(\mathrm{n}=100)$

\begin{tabular}{|c|c|}
\hline Age (years) & $47 \pm 12.7$ \\
\hline Age $(\geq 40$ years $)$ & $63(63)$ \\
\hline Gender (male) & $49(49)$ \\
\hline Tobacco smoking & $30(30)$ \\
\hline \multicolumn{2}{|l|}{ Marital status } \\
\hline Single & $12(12)$ \\
\hline Married & $70(70)$ \\
\hline Divorced & $4(4)$ \\
\hline Widow/widower & $14(4)$ \\
\hline \multicolumn{2}{|l|}{ Employment status } \\
\hline Yes & $81(81)$ \\
\hline No & $4(4)$ \\
\hline Retired & $15(15)$ \\
\hline \multicolumn{2}{|l|}{ Type of IBS } \\
\hline Constipation predominant & $45(45)$ \\
\hline Diarrhea predominant & $23(23)$ \\
\hline Alternating bowel habits & $32(32)$ \\
\hline \multicolumn{2}{|l|}{ Grade of IBS } \\
\hline Mild & $42(42)$ \\
\hline Moderate & $43(43)$ \\
\hline Severe & $15(15)$ \\
\hline BMI & $33 \pm 6.4$ \\
\hline Overweight & $20(20)$ \\
\hline Obesity & $69(69)$ \\
\hline Systemic hypertension & $14(14)$ \\
\hline $\mathrm{DM}$ & $32(32)$ \\
\hline Dyslipidemia & $29(29)$ \\
\hline MS & $19(19)$ \\
\hline NAFLD & $74(74)$ \\
\hline Mild & $17(17)$ \\
\hline Moderate & $33(33)$ \\
\hline Severe & $24(24)$ \\
\hline
\end{tabular}

IBS: Irritable Bowel Syndrome; n: Number; BMI: Body Mass Index; DM: Diabetes Mellitus; MS: Metabolic Syndrome; NAFLD: Nonalcoholic Fatty Liver Disease. Data Are Expressed as Frequency (Percentage), Except foror ageAnd BMI, which are expressed as mean \pm standard deviation.
Table 2. Factors associated with moderate/severe IBS among the study population $(\mathrm{n}=100)$

\begin{tabular}{|l|c|c|c|}
\hline & Mild IBS (n= 42) & $\begin{array}{c}\text { Moderate/severe } \\
\text { IBS (n= 58) }\end{array}$ & P \\
\hline Age ( $\geq 40$ years) & $20(47.6)$ & $43(74.1)$ & 0.072 \\
\hline Gender (male) & $15(35.7)$ & $34(58.6)$ & 0.059 \\
\hline Tobacco smoking & $7(16.7)$ & $23(39.7)$ & $0.014 *$ \\
\hline Marital status (except single) & $36(85.7)$ & $52(89.6)$ & 0.576 \\
\hline Employment & $36(85.7)$ & $45(77.6)$ & 0.428 \\
\hline Overweight/obesity & $36(86.7)$ & $53(91.4)$ & 0.514 \\
\hline Systemic hypertension & $5(11.9)$ & $9(15.5)$ & 0.398 \\
\hline DM & $5(11.9)$ & $27(46.6)$ & $0.001^{*}$ \\
\hline Dyslipidemia & $11(26.2)$ & $18(31)$ & 0.292 \\
\hline MS & $4(9.5)$ & $15(25.9)$ & $0.009 *$ \\
\hline NAFLD & $28(66.7)$ & $46(79.3)$ & 0.157 \\
\hline Moderate/severe NAFLD & $2(4.8)$ & $13(22.4)$ & $0.001^{*}$ \\
\hline
\end{tabular}

IBS: Irritable Bowel Syndrome; n: Number; DM: Diabetes Mellitus; NAFLD: Nonalcoholic Fatty Liver Disease. Data are expressed as frequency (Percentage). *Statistically significant.

Table 3. Predictors of moderate/severe IBS among the study population $(n=100)$

\begin{tabular}{|l|c|c|c|}
\hline & OR & $\mathbf{9 5 \%}$ CI & $\boldsymbol{P}$ \\
\hline MS & 3.1 & $1.8-54.6$ & $0.011^{*}$ \\
\hline Moderate/severe NAFLD (grade II) & 2.4 & $1.3-62.7$ & $0.026^{*}$ \\
\hline
\end{tabular}

IBS: irritable bowel syndrome; n: number; OR: odds ratio; CI: confidence interval; MS metabolic syndrome; NAFLD: nonalcoholic fatty liver disease. * Statistically significant.

among the patients with IBS and to uncover the association between moderate/severe NAFLD and moderate/severe IBS, possibly through the induction of an inflammatory state. In addition to moderate/severe NAFLD, MS was also an independent predictor of moderate/severe IBS among our study population.

The prevalence of NAFLD among our study population with IBS $(74 \%)$ is higher than the average reported prevalence rate among the general population [8]. This may be attributed to the exceptionally high prevalence of overweight/obesity (89\%) among our study population.

The low-grade inflammatory state among the patients with IBS can represent the contact point with NAFLD [14]. Physiologically, the intestinal mucosa harbors more immune cells compared to other tissues, due to its anatomical and functional characteristics as one of the barriers against micro-organisms [15]. These immune cells can be more abundant when the gut is irritated as in the case of IBS, as reported by previous studies [16,17]. Comparing patients with inflammatory bowel disease accompanied with IBS symptoms to those without such symptoms, it was found that the levels of calprotectin are significantly higher [18]. Calprotectin level reflects the inflammatory state as it is a calcium-binding protein produced by phagocytes with proinflammatory activity [19]. Such findings suggest that IBS can be regarded as an inflammatory disease. This may explain the impact of NAFLD on IBS, independent of the presence of MS among our study population with IBS.

This association may be related to the dysregulation of intestinal microbiota among patients with NAFLD responsible for more fat storage and steatosis -due to defective fermentation of polysaccharides to monosaccharide and impaired metabolism of short chain fatty acids[20] can contribute to the development of IBS, through the modulation of intestinal motility and sensitivity [21]. The NAFLD-related dysregulation of gut microbiota can lead to defect of the intestinal barrier function [22] and impaired intestinal immune tolerance [23]. In addition, NAFLD-related overexpression of toll-like receptors is a potential way of inducing cytokines release among the patients with IBS [24]. 
Among our study population with IBS, MS was an independent predictor of moderate/severe IBS in addition to moderate/severe NAFLD. Nonalcoholic fatty liver disease-associated MS yields a chronic inflammatory state, which can be systemic or local (hepatic) [25]. A previous study reported a significant association between IBS and MS [26].

Although our study aim was to investigate the prevalence of NAFLD among patients with IBS, the presence of a control group (subjects without IBS) would have been a valuable addition to explore the association between NAFLD and the development of IBS. The lack of laboratory confirmation of insulin resistance and relying on its clinical diagnosis of MS is considered a limitation of our study. In addition, we relied on abdominal ultrasonography for grading of liver steatosis without performing magnetic resonance imaging. We could not exclude the psychologic element as a confounder; the study patients were not subjected to psychologic assessment. However, it is the first study, as far as we know, to explore the impact of NAFLD on the severity of IBS.

\section{Conclusion}

In conclusion, we detected a high prevalence rate of NAFLD among patients with IBS. The predictors of moderate/severe IBS among our study patients were moderate/severe NAFLD and MS. Further studies including normal subjects without IBS are needed to explore the role of NAFLD in the development of IBS.

\section{References}

1. Drossman DA (2006) The functional gastrointestinal disorders and the Rome III process. Gastroenterology 130:1377-1390. [Crossref]

2. Quigley EM, Fried M, Gwee KA, Khalif I, Hungin AP, et al. (2016) World Gastroenterology Organisation Global Guidelines Irritable Bowel Syndrome: A Global Perspective Update September 2015. J Clin Gastroenterol 50: 704-713. [Crossref]

3. Occhipinti K, Smith JW (2012) Irritable bowel syndrome: a review and update. Clin Colon Rectal Surg 25: 46-52. [Crossref]

4. Hahn BA, Kirchdoerfer LJ, Fullerton S, Mayer E (1997) Patient-perceived severity of irritable bowel syndrome in relation to symptoms, health resource utilization and quality of life. Aliment Pharmacol Ther 11: 553-559. [Crossref]

5. Delgado-Aros S, Locke GR 3rd, Camilleri M, Talley NJ, Fett S, et al. (2004) Obesity is associated with increased risk of gastrointestinal symptoms: a population-based study. Am J Gastroenterol 99: 1801-1806. [Crossref]

6. Talley NJ, Quan C, Jones MP, Horowitz M (2004) Association of upper and lower gastrointestinal tract symptoms with body mass index in an Australian cohort. Neurogastroenterol Motil 16: 413-419. [Crossref]

7. Adams LA, Angulo P (2005) Recent concepts in non-alcoholic fatty liver disease Diabet Med 22: 1129-1133. [Crossref]

8. Ryan CK, Johnson LA, Germin BI, Marcos A (2002) One hundred consecutive hepatic biopsies in the workup of living donors for right lobe liver transplantation. Liver Transpl 8: 1114-1122. [Crossref]
9. Larter CZ, Chitturi S, Heydet D, Farrell GC (2010) A fresh look at NASH pathogenesis. Part 1: the metabolic movers. J Gastroenterol Hepatol 25: 672-690. [Crossref]

10. Tilg H, Diehl AM (2000) Cytokines in alcoholic and nonalcoholic steatohepatitis. $N$ Engl J Med 343: 1467-1476. [Crossref]

11. Feldstein AE, Werneburg NW, Canbay A, Guicciardi ME, Bronk SF, et al. (2004) Free fatty acids promote hepatic lipotoxicity by stimulating TNF-alpha expression via a lysosomal pathway. Hepatology 40:185-194. [Crossref]

12. Saadeh S, Younossi ZM, Remer EM, Gramlich T, Ong JP, et al. (2002) The utility of radiological imaging in nonalcoholic fatty liver disease. Gastroenterology 123: 745750. [Crossref]

13. Expert Panel on Detection, Evaluation, and Treatment of High Blood Cholesterol in Adults (2001) Executive Summary of The Third Report of The National Cholesterol Education Program (NCEP) Expert Panel on Detection, Evaluation, And Treatment of High Blood Cholesterol In Adults (Adult Treatment Panel III) JAMA 285: 2486-2497. [Crossref]

14. Scalera A, Di Minno MN, Tarantino G (2013) What does irritable bowel syndrome share with non-alcoholic fatty liver disease? World J Gastroenterol 19: 5402-520. [Crossref]

15. Furness JB, Kunze WA, Clerc N (1999) Nutrient tasting and signaling mechanisms in the gut. II. The intestine as a sensory organ: neural, endocrine, and immune responses. Am J Physiol 277: G922-928. [Crossref]

16. Dunlop SP, Hebden J, Campbell E, Naesdal J, Olbe L, et al. (2006) Abnormal intestina permeability in subgroups of diarrhea-predominant irritable bowel syndromes. $\mathrm{Am} \mathrm{J}$ Gastroenterol 101: 1288-1294. [Crossref]

17. Chadwick VS, Chen W, Shu D, Paulus B, Bethwaite P, et al. (2002) Activation of the mucosal immune system in irritable bowel syndrome. Gastroenterology 122: 17781783. [Crossref]

18. Grad C, David L, Portincasa P, Dumitrascu DL (2012) Diagnostic value of calprotectin in irritable bowel syndrome and in inflammatory bowel disease. Rom J Intern Med 50: 3-6. [Crossref]

19. Roth J, Vogl T, Sorg C, Sunderkötter C (2003) Phagocyte-specific S100 proteins: a novel group of proinflammatory molecules. Trends Immunol 24: 155-158. [Crossref]

20. Turnbaugh PJ, Ley RE, Mahowald MA, Magrini V, Mardis ER, et al. (2006) An obesity-associated gut microbiome with increased capacity for energy harvest. Nature 444: 1027-1031. [Crossref]

21. Collins SM, Denou E, Verdu EF, Bercik P (2009) The putative role of the intestinal microbiota in the irritable bowel syndrome. Dig Liver Dis 41: 850-853. [Crossref]

22. Tappenden KA, Deutsch AS (2007) The physiological relevance of the intestina microbiota--contributions to human health. J Am Coll Nutr 26: 679S-683S. [Crossref]

23. Gigante G, Tortora A, Ianiro G, Ojetti V, Purchiaroni F, et al. (2011) Role of gut microbiota in food tolerance and allergies. Dig Dis 29: 540-549. [Crossref]

24. Brint EK, MacSharry J, Fanning A, Shanahan F, Quigley EM (2011) Differential expression of toll-like receptors in patients with irritable bowel syndrome. $\mathrm{Am} \mathrm{J}$ Gastroenterol 106: 329-336. [Crossref]

25. Tilg H, Moschen AR (2010) Evolution of inflammation in nonalcoholic fatty liver disease: the multiple parallel hits hypothesis. Hepatology 52: 1836-1846. [Crossref]

26. Guo Y, Niu K, Momma H, Kobayashi Y, Chujo M, et al. (2014) Irritable bowel syndrome is positively related to metabolic syndrome: a population-based cross-sectional study. PLoS One 9: e112289. [Crossref]

Copyright: (C2018 Hasanain AFA. This is an open-access article distributed under the terms of the Creative Commons Attribution License, which permits unrestricted use, distribution, and reproduction in any medium, provided the original author and source are credited. 